\section{The promise of psychiatric enviromics}

\author{
JAMES C. ANTHONY
}

This is a special and exciting time for epidemiologists and psychiatrists who study the causes of mental and behavioural disturbances and the means of their prevention. On one flank, we have the fast-paced Human Genome Project (HGP). The 'genome' has been defined as the total ensemble of genetic material for a form of life, or as the minimal complete complement of DNA molecules and chromosomes. Thanks to automation and development of polymerase chain reaction (PCR) methods since the mid-1980s, this initiative soon will yield a complete genetics database that maps and sequences the complete human genome, possibly 3 billion base pairs making up some 20000 or more gene loci. This step in the HGP will be complete in 2005 or before (Collins et al, 1998).

Even before completion of the HGP, an emerging 'Human Proteome Initiative' (HPI) has started to capture the attention of molecular biologists, geneticists and combinatorial chemists. The term 'proteome' (or 'proteom') has been used to describe systematic identification of the total protein complement of the genome. It also can be defined as a complete database of protein expression - not just expression in the form of a single protein, but also in the form of protein systems, such as systems of inflammation and repair after injury. Proteomics marries information about DNA sequence with RNA expression and with expressed protein data (Humphery-Smith et al, 1997).

In tandem with basic proteomics research, there is an applied agenda for combinatorial chemists, pharmacologists and the pharmaceutical and nutraceutical research industries. A goal of this agenda is knowledge of a full profile of genes upregulated or downregulated by specific drug compounds (or by other environmental exposures). Achieving this goal will amount to a full annotation of pharmacological (or nutraceutical) impact across genome and protein systems, a massive task.
Paul Negulescu (personal communication, 1998) has appraised the magnitude of the task: imagine an array of just 50 geneexpressed protein targets and a relatively small set of 500000 chemical compounds that might have upregulating or downregulating activity; these would require 250 million target-compound assays. At the research and development laboratories current throughput of about 6000 assays per day, this screening task would take 10 years. If the assay throughput could be increased to 100000 assays per day, the task would take less than a year, with impressive implications for revenues from product research and development (P. A. Negulescu, personal communication, 1998).

Consider the value to the pharmaceutical industry of such a reduction in the lead time for identifying a target neuropsychiatric compound, such as a novel drug along the lines of anti-inflammatory cyclo-oxygenase inhibitors (e.g. ibuprofen and aspirin). These compounds have been found in some studies to have inverse associations with occurrence of Alzheimer's-type dementia. These inverse associations are of special interest to investigators of this disorder because these compounds might decrease efficiency of synaptic response or post-synaptic signalling in cells with $\mathrm{N}$ methyl-D-aspartate (NMDA)-type glutamate receptors, which are vulnerable to excitotoxic death under conditions of excessive stimulation (Breitner, 1996). For developing of a new cyclo-oxygenase inhibitor to prevent Alzheimer's disease, as for other more general innovations described by Negulescu (P. A. Negulescu, personal communication, 1998), millions of dollars of earlier revenues might be gained if the race towards application were achieved even 6-10 months faster than today's pace of research and development allows.

With advances such as these, we can count upon genomics and proteomics for major discoveries that will transform the practice of psychiatry and psychiatric epidemiology over the coming decades; but neither genomics nor proteomics encompass the scientific agenda required to discover the full interplay of environment with genome and proteome. As specified at present, genomics and proteomics allow for environmental interplay within explanatory models of human health and behaviour, but do not include a deliberate strategy of search for aspects of the human environment that can be modified to alter the risks we inherit by virtue of our genes 
(Collins et al, 1998; Humphery-Smith et al, 1997). The human genome dwells within environment, determines environment, and its expression is shaped by environment (Anthony et al, 1995; Brakefield \& French, 1995; Brakefield et al, 1996; Kendler \& Prescott, 1998). These are topics for epidemiological research.

Beyond the microscopic orientation of genomics and proteomics, an epidemiologist's search for modifiable aspects of human environment can encompass social and experiential processes and conditions that fall outside the conventional boundaries of molecular biology, genetics and combinatorial chemistry (Anthony et al, 1995). Beyond these boundaries lie adverse conditions, such as infection or chemical exposure during gestation, nutritional deficiencies, and severe neglect and abuse during the childhood years; or more salubrious conditions and processes, such as effective parental supervision and monitoring to reduce affiliation with delinquent peers, high-quality schooling toward literacy and numeracy and more salubrious conditions of the psychosocial work environment. With modern multi-level statistics, these conditions and processes may be discovered to depend upon social cohesion, collective efficacy or other aspects of locally shared environments (Sampson et al, 1997; Petronis \& Anthony, 2000).

The search is on for individual or group characteristics, conditions and processes that contribute to the onset of mental disorders or influence their course - net of, in combination with, or in addition to the influence of genes and their protein expressions. As described elsewhere (Anthony et al, 1995), this search can become a 'human envirome project' for psychiatric epidemiology, an initiative that seeks the total ensemble of environments, both current and in earlier life, that affect the occurrence of mental and behavioural disturbances. Complementing psychiatric research based on genomics and proteomics, 'psychiatric enviromics' can focus on aspects of environment that affect mental health and behaviour.

Psychiatric 'enviromics' is not just old psychiatric genetics with a new label. In its present form psychiatric genetics is oriented towards mapping candidate genes for mental disorders, as well as searching for candidate symptoms, endophenotypes or vulnerability traits that are best regarded as expressions of genes, singly or in combination (see, for example, Owen \&
Craddock, 1996; Leboyer et al, 1998). In time, psychiatric genetics will give way to the more broad-based proteomics initiative and to investigations in combinatorial chemistry mentioned above.

Psychiatric enviromics can be planned as a deliberately complementary search for specific environments or environmental processes and conditions that promote mental health and reduce the occurrence of psychiatric disturbances. Subsets of the psychiatric envirome will be discovered to have functional importance precisely because specific environmental conditions or processes will reduce, amplify, or otherwise modulate the expression of specific genes or multiple gene interactions at identifiable periods of life-span development. In these circumstances, the indicated intervention might well be developed as a pharmaceutical or nutritional supplement.

Nevertheless, any plan for psychiatric enviromics must be broad enough to accommodate salubrious environmental conditions and processes that have functional importance but lack specificity of action with respect to gene expression. This argues for a multi-disciplinary range that encompasses medicinal chemistry, pharmacology, pharmacogenetics and the corresponding nutrition sciences, and also reaches toward human ecology, sociology, social relations, social psychology and their traditional intersections within social psychiatry.

One starting point for psychiatric enviromics is the catalogue of mental health-damaging environmental exposures and conditions compiled by Ernest Gruenberg and colleagues within the American Public Health Association (APHA) for a now-outdated manual entitled Mental Disorders: A Guide to Prevention and Control, last published in 1962 (American Public Health Association, 1962). A map of the psychiatric envirome and genome could be developed through an international collaboration to bring this manual up-to-date. These complementary maps could shed new light on the expression of specific genes and gene interactions that influence mental health, as well as on the complementary influences of environmental conditions and processes. If organised in relation to specific environments encountered during discrete periods of life span development, the process of mapping the psychiatric envirome would throw into relief gaps in our present knowledge, as well as the forms of evidence and new plans of investigation required to fill these gaps.

The APHA manual described above was designed to complement the APHA manual on control of communicable diseases in humans, and was intended to be used in the same way that public health workers used the communicable disease manual. It would guide them in identification of cases of mental disorders, in efforts to intervene quickly once a case was recognised and to prevent occurrence of other cases when possible.

The APHA manual on mental disorders has not been updated in any formal way since 1962. Some of the known causes of mental disorders it lists have been replaced in importance by others. For example, bromism is no longer is as important as it once was; other medicines have taken the place of bromides in the pharmacopoeia. Much has been learned since 1962, however, not only with respect to pharmacology, but also with respect to genetic causes of psychiatric syndromes, communicable or infective agents such as the dinoflagellate Pfisteria, the Borna virus, human immunodeficiency virus (HIV) infection and the suspected prion disease manifested in connection with bovine encephalopathies. We have toxic exposures that Gruenberg and his colleagues never knew about, though they might have dreamed of 'designer drugs' such as MDMA (methylenedioxymethamphetamine) and other mixed stimulant-hallucinogens, the toxic consequences of maternal smoking and drinking, or the effects of new nerve gases.

Advances also have been made along more psychosocial lines. Evidence about the financial strains of unemployment has mounted, and we have new leads for possibly salubrious improvements in the workplace. Early research on combat fatigue and the shell-shock syndrome has developed into a major enterprise to sort out which traumatic events might be most harmful to human mental health. I could go on in this vein, but fortunately Eaton and Harrison (1998) have done so in a recent review of contemporary research.

The first step in the proposed plan for psychiatric enviromics is to consolidate and to catalogue what we now can say with confidence about environmental conditions and processes that impinge on human mental health. The manual on mental disorders created by Gruenberg's committee serves as a point of departure, but we can 
now be more specific about the psychiatric syndromes known to have the strongest associations with each environmental condition or process under scrutiny. This endeavour would be suited to an international organisation such as the International Federation of Psychiatric Epidemiology, much as the APHA sponsored the work of Gruenberg's committee, who could provide the necessary support for meetings, correspondence and administration.

This initial step in psychiatric enviromics would allow us to chart the next incremental step, probably involving consolidation of information about the genome, the proteome and how the expression of gene interacts with the envirome. That is, within a decade, multiple candidate genes and gene combinations would be identified in association with major psychiatric syndromes or newly identified endophenotypes, as well as some of the less prominent syndromes such as cannabis dependence (see, for example, Kendler \& Prescott, 1998). However, no one is willing to place large bets on the 'one gene-one syndrome' model, and the incomplete penetrance of gene expression with respect to psychiatric syndromes generally represents an opportunity to probe for environmental conditions and processes that influence the observed variation - in tandem with an opportunity to probe for effect-modifying genes and gene combinations.

The relative success and speed of inquiry with respect to genetic sources of variation must give the geneticists the upper hand in this search. Almost anyone who has tried seriously to measure human exposure to environmental conditions and processes has faced the humiliating experience of having to rely upon retrospective self-reports or proxy informant reports of uncertain reliability and validity, or upon representations of the environmental exposure that are arguably more objective and reliable, but not necessarily more accurate or valid.

Our work on Alzheimer's disease in Australia and in Utah provides a case in point (Broe et al, 1990). The retrospective information about exposure to analgesic and anti-inflammatory medicines provides no more than weak evidence of an inverse association between these medicines and occurrence of Alzheimer's-type dementia syndromes. Looking into medicine cabinets has helped us to confirm exposures, and provides more potent inverse associations, but this representation leaves much to be desired in relation to completeness of the dosing history. Future work along these lines must include better measurement of the environment predicted to interact with gene expression - not only a more reliable measurement, but also a more valid one.

Whether we shall be able to harness molecular probes and PCR techniques to improve our measurement of biological exposures for the purpose of psychiatric research remains to be seen. A promising methodological development involves the measurement of PCR-amplified signals of exposure to environmental toxins and drugs, in the form of DNA adducts, which ultimately might promote carcinogenesis. Progress has been made in the use of such DNA adducts as a durable marker of tobacco exposure. Even if well-calibrated measures of the 'social envirome' (Eaton \& Harrison, 1998) are unlikely to be available for some time, substantial progress in improving methods of assessment is attested to by several of the contributions to this supplement. It is not unreasonable to hope that more precise, focused analysis of gene-proteomeenvirome relationships will serve to extend our causal understanding of mental disorders.

In summary, psychiatric enviromics research requires us to choose our problems carefully, favouring gene expression that is malleable by environment, and choosing envirome variables that can be reliably measured, returning to less easily soluble problems as progress is made - much as the combinatorial chemists are choosing to start their work with polypeptides having seven to nine amino acids because these compounds are overrepresented among currently known biologically active molecules with receptor activity.

However, a word of caution is needed, as Bill Eaton, Scott Henderson and I mentioned when we first wrote about the human envirome initiative (Anthony et al, 1995). Namely, the case of this line of research is toward well-specified disease targets. As Medawar might have said, this is appropriate because these look like the most soluble problems at present. None the less, our choice of these specific targets might leave behind the less discrete and more prevalent psychiatric disturbances and associated impairments and disabilities. One of the challenges of the future will be to bring genomics, proteomics and enviromics face to face with these more ubiquitous forms of psychiatric suffering, encountered not only in the technologically advanced nations where there is sufficient material wealth to entertain advanced concepts of gene therapy and to afford pharmaceutical and nutraceutical agents, but also in the emerging market economies where the mental health of billions must rely upon something else.

\section{ACKNOWLEDGEMENTS}

The author wishes to acknowledge travel support from the International Federation of Psychiatric Epidemiology as well as a grant award from the US National Institutes of Health, National Institute on Drug Abuse (IROIDA09897)

\section{REFERENCES}

American Public Health Association (1962) Menta Disorders: A Guide to Prevention and Control.Washington, DC: APHA

Anthony, J. C., Eaton, W. W. \& Henderson, A. S. (1995) Looking to the future in psychiatric epidemiology. Epidemiologic Reviews, I7, 240-242.

Brakefield, P. M. \& French, V. (1995) Eyespot development on butterfly wings: the epidermal response to damage. Developmental Biology, 168 98-III.

_, Gates, J., Keys, D., et al (1996) Development, plasticity and evolution of butterfly eyespot patterns. Nature, 384, 236-242.

Breitner, J. C. S. (1996) Inflammatory processes and antiinflammatory drugs in Alzheimer's disease: a current appraisal. Neurobiology of Aging, 17 789-794.

Broe, G. A., Henderson, A. S., Creasey, H., et al (1990) A case-control study of Alzheimer's disease in Australia. Neurology, 40, 1698-1707.

Collins, F. S., Patrinos, A., Jordan, E., et al (1998) New goals for the US Human Genome Project: 1998 2003. Science, 282, 682-689.

Eaton, W. W. \& Harrison, G. (1998) Epidemiology and social aspects of the human envirome (editorial). Current Opinion in Psychiatry, II, 165-168.

Humphery-Smith, I., Cordwell, S. J. \& Blackstock, W. P. (1997) Proteome research: complementarity and limitations with respect to the RNA and DNA worlds. Electrophoresis, 18, 1217-1242.

Kendler, K. S. \& Prescott, C. A. (1998) Cannabis use, abuse, and dependence in a population-based sample of female twins. American Journal of Psychiatry, I55, 1016-1022. 
Leboyer, M., Bellivier, F., Nosten-Bertrand, M. et al (1998) Psychiatric genetics: search for phenotypes. Trends in Neurosciences, $\mathbf{2}$ $102-105$

Owen, M. J. \& Craddock, N. (1996) Modern molecular genetic approaches to complex traits: implications for psychiatric disorders. Molecular Psychiatry, I, 21-26.

JAMES C. ANTHONY, PhD, Department of Mental Hygiene, School of Hygiene and Public Health, Johns Hopkins University, Baltimore, Maryland, USA. E-mail: janthony@jhu.edu

Petronis, K. R. \& Anthony, J. C. (2000) Perceived risk of cocaine use and experience: do they cluster within US neighborhoods and cities? Drug and Alcohol Dependence, 57, $183-192$.
Sampson, R. J., Raudenbush, S.W. \& Earis, F. (1997) Neighborhoods and violent crime: a multilevel study of collective efficacy. Science, 277, $918-924$ 